\title{
Die FMH erschliesst mit der Plattform HIN den Nutzen der digitalen Zukunft!
}

Urs Stoffel

Mitglied des Zentralvorstands FMH, Verantwortlicher Ressort eHealth - Sicherheitsinfrastruktur und VR-Präsident HIN

\section{Vertrauen schaffen im elektronischen Datenaustausch und in der digitalen Kommunikation}

Zweifellos stehen wir erst am Anfang der Umsetzung der eHealth-Strategie des Bundes. Noch hegen Ärzteschaft und Bevölkerung recht grosse Vorbehalte gegenüber den Nutzen und den Gefahren dieser neuen, innovativen Technologie. Unser Ziel muss deshalb sein, die Ängste vor eHealth abzubauen und sowohl die Patientinnen und Patienten als auch die Ärzteschaft vom Nutzen von eHealth zu überzeugen.

\section{Pragmatische Lösungen mit Nutzen sind gefragt, die nah bei der Basis und bei den Patienten sind.}

Die FMH hat die vitale Bedeutung einer gesicherten Kommunikation im elektronischen Datenaustausch bereits früh erkannt und dafür vor 16 Jahren die HIN-Plattform gegründet. Damit wurde ein essentieller Baustein gelegt, um Vertrauen und Vertraulichkeit im Netz sicherzustellen. Die Werkzeuge von HIN werden heute tagtäglich von Tausenden Ärztinnen und Ärzten genutzt und finden weit über ihre Grenzen hinaus grosse Beachtung. anderer europäischer Länder lernen und wo nötig Gegensteuer geben.

\section{HIN-Plattform baut Angebot aus und investiert in zukunftsgerichtete und innovative Technologien}

Die FMH wird sich verstärkt und fokussiert dafür einsetzen, die bestehende HIN-Plattform als wichtiges Werkzeug zur sicheren Nutzung von eHealth auszubauen. Das Dienstleistungsangebot für die Mitglieder der «HIN Community» wird sukzessive mit dem Ziel erweitert, einen klaren Mehrwert für die HINAbonnenten zu schaffen. In enger Abstimmung mit der FMH entwickelt HIN moderne Werkzeuge, die den Alltag der Ärztinnen und Ärzte erleichtern und ein mobiles Arbeiten ermöglichen. Die Vernetzung und der Datenaustausch sind für die FMH zentrale Anliegen - und zwar ohne Einsatz von teuren transaktionsabhängigen Diensten, sondern mit ärzteeigenen Institutionen und Plattformen.

\section{Nutzen für Patienten und Ärzteschaft im Zentrum}

Es gibt bereits erfolgreiche Beispiele für einen nutzbringenden Einsatz der eHealth-Technologie. Die online durchführbaren medizinischen Fahrtauglichkeitskontrollen der Strassenverkehrsämter (emedko), der sichere Versand von Austrittsberichten und ein

\section{Die bestehende HIN-Plattform wird als wichtiges Werkzeug zur sicheren Nutzung von eHealth ausgebaut.}

\section{Korrespondenz: \\ Dr. med. Urs Stoffel \\ FMH \\ Elfenstrasse 18 \\ CH-3000 Bern 15 \\ Tel. 0313591111 \\ Fax 0313591112}

kommunikation[at]fmh.ch
Dank der HIN-Plattform werden der Datenschutz und das Arztgeheimnis auch in der digitalen Kommunikation respektiert. Zudem wird die Identität der jeweiligen Kommunikationspartner vor der Benützung der elektronischen Medien überprüft und garantiert. Dies schafft Vertrauen in die neuen Online-Technologien.

Dennoch klafft nach wie vor eine grosse Lücke zwischen den eHealth-Wunschvorstellungen und der Realität. Deshalb sind pragmatische Lösungen mit Nutzen gefragt, die nah bei der Basis und bei den Patienten sind. Hier müssen wir auch ein wachsames Auge auf die Entwicklungen im nahen Ausland in diesem Bereich haben. Wir müssen aus den Fehlern einfacher, sicherer Zugriff auf Patientendossiers im Spital, beispielsweise in den Zürcher Stadtspitälern, sind praktische Anwendungen von eHealth. Nicht zuletzt steht heute auch die Möglichkeit einer sicheren und einfachen Kommunikation mit Patienten per E-Mail (Secure Mail GLOBAL) zur Verfügung. All diese Lösungen wurden auf Basis der HIN-Plattform entwickelt.

Es gibt noch viel zu tun ... Wir von der FMH wollen diese Aufgabe zusammen mit HIN anpacken und die bedarfsorientierte Weiterentwicklung der geeigneten Werkzeuge zur Umsetzung von eHealth zum Nutzen für Patienten und Ärzteschaft in eine digitale Zukunft unterstützen und fördern. 